EESTI NSV TEADUSTE AKADEEMIA TOIMETISED.

FOUSIKA * MATEMAATIKA

ИЗВЕСТИЯ АКАДЕМИИ НАУК ЭСТОНСКОИ ССР. ФИЗНКА * МАТЕМАТИКА

PROCEEDINGS OF THE ACADEMY OF SCIENCES OF THE ESTONIAN SSR.

PHYSICS * MATHEMATICS

$1985,34,2$

удк 6.013 .42

H. ВЕКСЛЕР, Ю. КАРТАШОВ

\title{
РАССЕЯНИЕ АКУСТИЧЕСКИХ ВОЛН КРУГОВЫМ ЖИДКИМ ЦИЛИНДРОМ, СОДЕРЖАЩИМ ЖИДКИЙ ЗАПОЛНИТЕЛЬ
}

\author{
(Представил Н. Алумяз)
}

Представляются результаты расчета частотной и временной зависимостей акустического давления, рассеянного назад круговым жидким цилиндром с коаксиальным жидким заполнителем. Дается объяснение резонансной природы процесса рассеяния. Предлагается формула для расчета расходимости волн при переотражении в цилиндрическом слое. Приводится сравнение результатов с полученными в предельном плоском случае.

\section{1. Постановка и решение задачи}

Пусть в безграничной идеальной сжимаемой жидкости имеется жидкий цилиндр, содержащий коаксиальный жидкий заполнитель. На цилиндр падает акустический волновой пакет давления, плоский фронт которого параллелен образующим цилиндра. Требуется рассчитать вторичное рассеянное - поле акустического давления в жидкости и дать его физическую интерпретацию.

Трудно представить как в реальности можно организовать рассеяние волн жидким цилиндром с коаксиальным жидким заполнителем. Фактически этого и не требуется. По существу, рассматриваемая задача является модельной по отношению к задаче о рассеянии упругим цилиндром с упругим заполнителем. При рассеянии упругим телом, когда имеется угол между нормалью к поверхности контакта и направлением распространения волны, приходящей к этой поверхности, при каждом отражении (прохождении) происходит трансформация продольной волны в поперечную (и наоборот). Из-за этого быстро растет число типов волн, которые надо исследовать, а их амплитуда, соответственно, падает. В случае предельной - жидкой - задачи такой трансформации не происходит и поэтому число возбудившихся типов волн здесь меньше. Слово жидкий применительно к цилиндру и его заполнителю понимается не как состояние, а означает, что в твердом упругом материале искусственно (намеренно) блокировано распространение поперечных волн.

Как известно $\left[{ }^{1}\right]$, решение задачи в пространстве интегрального преобразования Фурье по времени имеет вид

$$
p_{s}^{F}(x)=p_{0} \sum_{n=0}^{\infty} X_{7 n} H_{n}^{(1)}(x r) \cos n \theta, \quad p_{0}=p_{\star} \exp (i x) g^{F}(x) .
$$

Здесь $p_{s}^{F}$ - изображение Фурье акустического давления в точке наблюдения $(r, \theta), x=k a-$ волновой радиус в жидкости, окружающей цилиндр, $a$ - наружный радиус цилиндра, $b$ - наружный радиус заполнителя, $r=R / a-$ безразмерная радиальная координата, $\theta-$ полярный угол', $p_{*}$ - амплитуда давления в падающем волновом пакете, $g^{F}-$ изображение Фурье зависимости изменения давления в падающем вол- 
новом пакете, $H_{n}{ }^{(1)}$ - функция Ханкеля первого рода. Остальные величины в формуле (1) и ниже определены в [ $\left.{ }^{1}\right]$.

Для удобства представления результатов проведем нормировку

$$
\begin{gathered}
p^{F}(x)=p_{s}^{F}(x) / m g F(x), \quad m=p_{*}[r(2 \cos \gamma / \cos \alpha-1 / r)]^{-1 / 2} \\
p(\tau)=p_{s} / m, \quad \tau=t-(r-1) .
\end{gathered}
$$

Расчет выполним для точки наблюдения, расположенной на луче $\theta=0$, ориентируясь на наиболее часто встречающийся в практике случай, когда источник и приемник волн совмещены. Тогда $\theta=\alpha=\gamma=0$ и параметр $m$ получает значение

$$
m=p_{\star}[r(2-1 / r)]^{-1 / 2}, \quad r \geqslant 1 .
$$

Отсчет времени $\tau$ производится с момента, когда фиксированной точки наблюдения достигнет отраженная цилиндром волна.

Модуль функции $p^{F}(x)$ будем называть частотной зависимостью, а $p(\tau)$ - временной зависимостью.

Назначим физические параметры задачи. Пусть в воде размещается жидкий цилиндр из армко-железа, заполненный водой. Примем

$$
\begin{gathered}
\mathrm{Q}=\mathrm{Q}_{10}=1 \cdot 10^{3} \mathrm{Kr} / \mathrm{M}^{3}, \quad c=c_{10}=1493 \mathrm{~m} / \mathrm{c}, \\
\mathrm{Q}_{1}=7,7 \cdot 10^{3} \mathrm{Kr} / \mathrm{m}^{3}, \quad c_{1}=5960 \mathrm{M} / \mathrm{c}, \quad h=1-b / a=1 / 8 . \\
r=10^{4}, \quad \theta=0 .
\end{gathered}
$$

На рисунке показана частотная зависимость давления, рассчитанная в широком диапазоне изменения волнового радиуса $x=0-200$ с шагом $l_{x}=1 / 256$. Из-за мелкого масштаба графика некоторые из экстремумов кривой частотной зависимости слились, а отдельные экстремумы стали неразличимыми.

\section{2. Анализ частотной зависимости}

Из рисунка видно, что частотная зависимость весьма изрезана и нерегулярна. Ее подробный анализ достаточно сложен. Здесь мы рассмотрим только основные свойства этой зависимости.

Частотную зависимость формируют волны, возникшие в следующих процессах: зеркальном отражении от наружной поверхности, переотражении, переизлучении и дифракции (в узком смысле).

Наша главная цель - промоделировать процесс рассеяния упругим телом. Возникающие же в рассматриваемой здесь предельной задаче переизлученные волны по своей природе имеют мало общего с однотипными волнами в случае упругого рассеивателя и поэтому не анализируются.

Качественно, дифрагированные волны ведут себя подобно одноименным волнам в случае «акустически жесткого» цилиндра. В освещенной области их вклад, вообще говоря, мал и заметен только при $x \leqslant 20$. Поэтому в этой задаче мы их не рассматриваем.

Зеркальное отражение. При $2 \leqslant x \leqslant 10$ для описания зеркально отраженной волны можно использовать приближенную формулу

$$
\begin{gathered}
p_{1}^{F}(x) \sim v \bar{p}_{1}(x), \quad v=(1-\zeta) /(1+\zeta), \quad \bar{p}_{1}(x)=1-i A_{1} x^{-1}-A_{2} x^{-2}, \\
A_{1}=11 / 16, \quad A_{2}=353 / 512,
\end{gathered}
$$

где $v$ - коэффициент отражения от плоской границы раздела двух жидких сред при нормальном падении волны из среды $\varrho, c$ в среду $\varrho_{1}, c_{1}$, а 

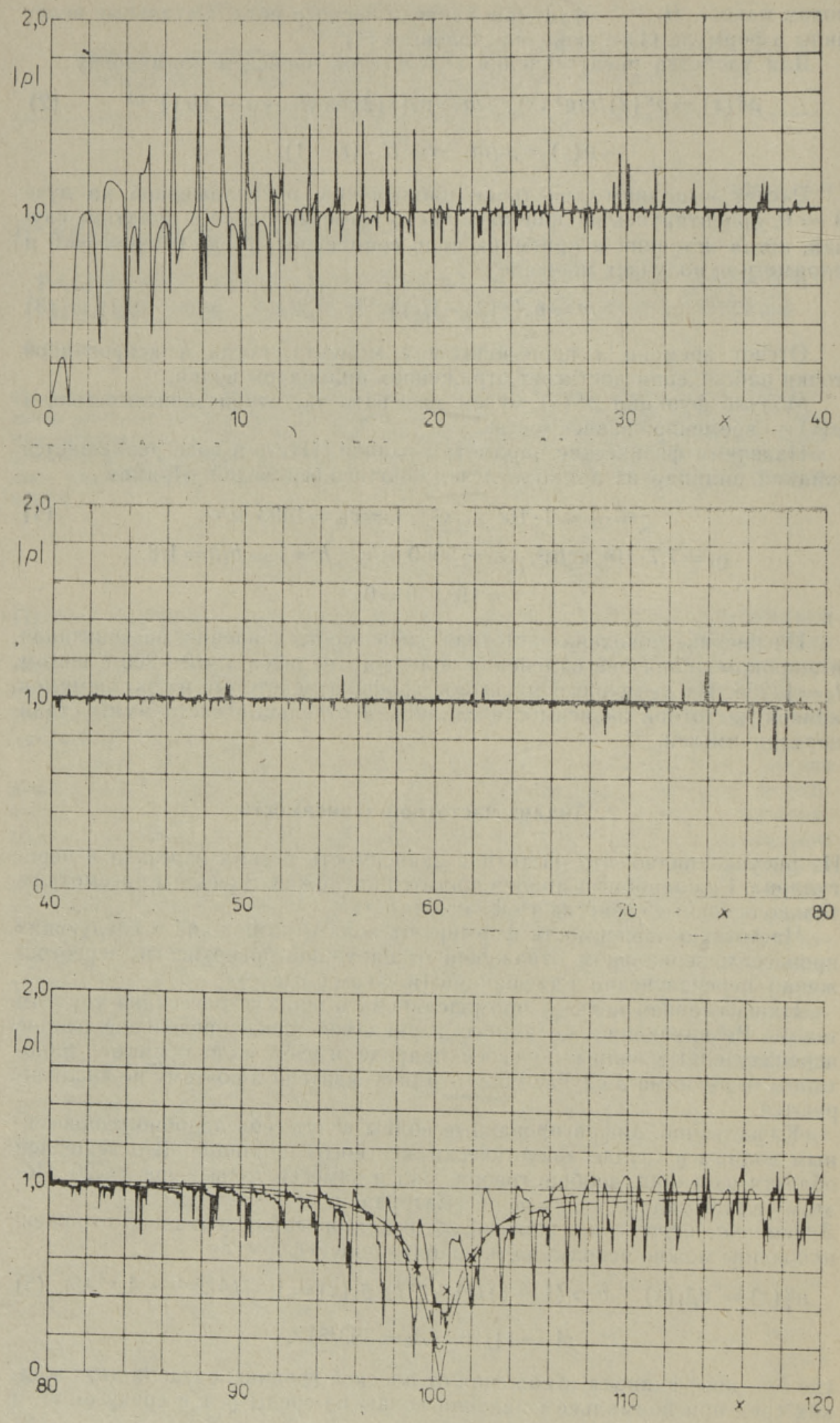

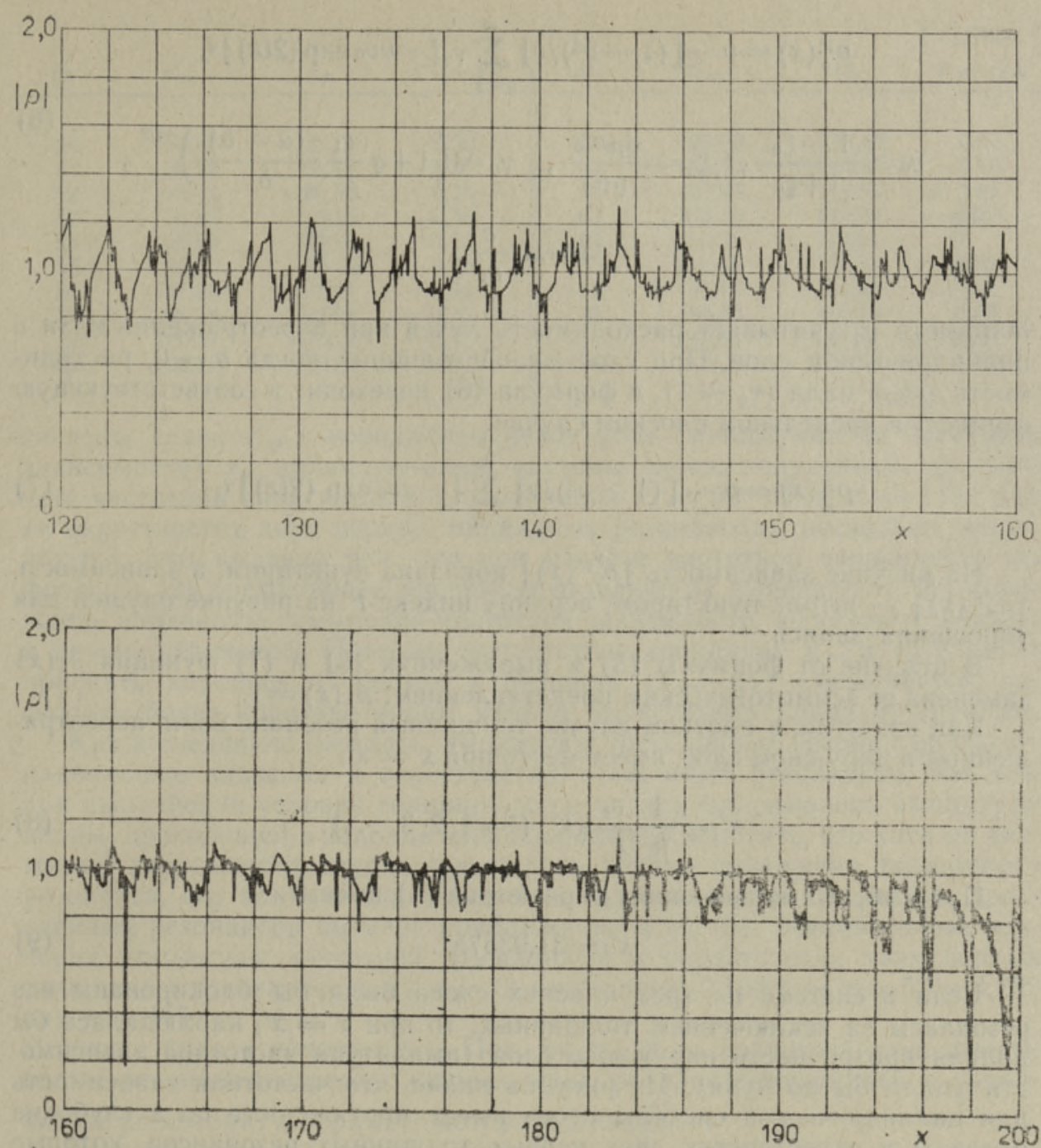

$\bar{p}_{1}$ - коротковолновоё приближение амплитуды волны, зеркально отраженной «акустически жестким» цилиндром $\left[{ }^{2}\right]$. При $x \geqslant 10$ амплитуду зеркально отраженной волны можно считать равной $v$. Мы использовали вместо цилиндрического коэффициента отражения плоский. Такая замена тем точнее, чем больше $x$.

Переотражение. Рассмотрение рисунка наталкивает на мысль, что в различных диапазонах $x$ можно выделить три доминирующих типа резонансов волн, переотражающихся по следующим трассам: первая - переотражение в наружной стенке; вторая - переотражение в трехслойной системе жидких слоев; третья - переотражение в жидком заполнителе вдоль квадрата со стороной $b \sqrt{2}\left[{ }^{3}\right]$.

Толщцнный резонанс. Используя интегральную формулу Кирхгоффа совместно с методом стационарной фазы, можно получить в коротковолновом приближении формулу для амплитуды давления в точке наблюдения $(r \gg 1, \theta=0)$, учитывающую зеркальное отражение от внешней поверхности цилиндра и переотраженные в наружном (ближайшем к источнику) слое в виде 


$$
\begin{gathered}
p_{0}^{\vec{F}}(x)=v-\left[\left(1-v^{2}\right) / v\right] \sum_{q=1}^{\infty} \gamma_{q}\left[-v v_{0} \exp (2 i z)\right]^{q}, \\
v_{0}=\frac{1-\zeta_{0}}{1+\zeta_{0}}, \quad \zeta_{0}=\frac{\varrho_{10} c_{10}}{\varrho_{1} c_{1}}, \sim \gamma_{q}=\left(1+q \frac{c_{1}}{c} \frac{(a-b)}{b}\right)^{-1 / 2}, \\
z=x h \frac{c}{c_{1}} .
\end{gathered}
$$

Параметр $\gamma_{q}$ учитывает расходимость лучей при переотражении волн в цилиндрическом слое. При слое малой толщины, когда $h \rightarrow 0$, расходимость лучей мала $\left(\gamma_{q} \rightarrow 1\right)$, и формула $(6)$ переходит в соответствующую формулу в предельном плоском случае

$$
p_{*}^{F}(x)=v-\left[\left(1-v^{2}\right) / v\right] \sum_{q=1}^{\infty}\left[-v v_{0} \exp (2 i z)\right]^{q} .
$$

На рисунке зависимость $\left|p_{0}^{F}(x)\right|$ показана пунктиром, а зависимость $\left|p_{\star} F(x)\right|-$ штрих-пунктиром; верхний индекс $F$ на рисунке опущен для упрощения записи.

В отличие от формулы (5) в выражениях (6) и $(7)$ функция $\bar{p}_{1}(x)$ заменена ее асимптотическим представлением: $\bar{p}_{1}(x)=1$.

Как известно, в плоском случае толщинный резонанс волн, переотраженных в наружном слое, имеет место при $x=x^{\prime}$ เ

$$
x^{\prime} l=\frac{1}{h} \frac{c_{1}}{c} \pi l \quad(l=1,2,3, \ldots) .
$$

При выбранных значениях параметров (4) имеем

$$
x^{\prime}=31,9357 \pi l \text {. }
$$

Если в системе из трех плоских слоев были бы блокированы все резонансы за исключением толщинных, то при $x=x_{l}^{\prime}$ наблюдалась бы полная прозрачность наружного слоя (амплитуда частотной зависимости упала бы до нуля). Из рисунка видно, что частотная зависимость для цилиндрической системы слоев имеет протяженные по $x$ глубокие провалы в окрестностях двух первых толщинных резонансов, которые при выбранных значениях параметров находятся на $x_{1}^{\prime}=100,33$ и $x_{2}^{\prime}=2 x_{1}^{\prime}$.

Трехслойный резонанс. В плоском случае он имеет место при

$$
x_{m}^{\prime}=m \Delta x_{m}^{\prime} \quad(m=1,2,3 ; \ldots),
$$

где шаг следования трехслойных резонансов $\Delta x_{m}^{\prime}$ находится из уравнения

$$
\Delta x_{m}^{\prime} \Delta t_{0}=2 \pi
$$

Здесь $\Delta t_{0}$ - безразмерное время, затраченное волной на пробег удвоенного пути вдоль диаметра цилиндра

$$
\Delta t_{0}=4\left[h / v^{p h}+(1-h) c / c_{10}\right],
$$

где $v^{p h}=c_{1} / c$ - относительная скорость распространения волны в цилиндрическом слое.

При выбранных параметрах (4) имеем

$$
\Delta t_{0}=3,625, \quad \Delta x_{m}^{\prime}=1,733 .
$$

Как видно из табл. 1, координаты трехслойных резонансов плоской 
Тิаблица 1

\begin{tabular}{|c|c|c|c|c|c|c|c|}
\hline$m$ & $x_{m}^{\prime}$ & $x_{m}$ & $x_{m}^{\prime}-x_{m}$ & $m$ & $x_{m}^{\prime}$ & $x_{m}$ & $x_{m}^{\prime}-x_{m}$ \\
\hline $\begin{array}{l}50 \\
51 \\
52 \\
53 \\
54 \\
55 \\
56 \\
57 \\
58 \\
59\end{array}$ & $\begin{array}{r}86,65 \\
88,38 \\
90,12 \\
91,85 \\
93,58 \\
95,32 \\
97,05 \\
98,78 \\
100,51 \\
102,25\end{array}$ & $\begin{array}{c}86,95 \\
88,44 \\
89,88 \\
92,25 \\
93,98 \\
95,16 \\
97,41 \\
98,98 \\
100,66 \\
101,95\end{array}$ & $\begin{array}{r}-0,30 \\
-0,06 \\
0,24 \\
-0,40 \\
-0,40 \\
0,16 \\
-0,36 \\
-0,20 \\
-0,15 \\
0,30\end{array}$ & $\begin{array}{r}60 \\
61 \\
62 \\
63 \\
64 \\
65 \\
112 \\
113 \\
114 \\
115\end{array}$ & $\begin{array}{c}103,98 \\
105,71 \\
107,45 \\
109,18 \\
110,91 \\
112,65 \\
194,10 \\
195,83 \\
197,56 \\
199,30\end{array}$ & $\begin{array}{l}103,53 \\
105,87 \\
107,55 \\
109,23 \\
110,91 \\
112,57 \\
194,06 \\
195,93 \\
197,54 \\
199,28\end{array}$ & $\begin{array}{c}0.45 \\
-0,16 \\
-0,10 \\
-0,05 \\
0 \\
0,08 \\
0,04 \\
-0,10 \\
0,02 \\
0,02\end{array}$ \\
\hline
\end{tabular}

системы слоев $x_{m}^{\prime}$ и координаты резонансов (минимумов на частотной зависимости) $x_{m}$ цилиндрической системы слоев, полученные при расчете частотной зависимости, для значений $m=50-65$ и $m=112-115$ (в окрестностях двух первых толщинных резонансов), несколько различаются. Это вызвано тем, что при расчете частотной зависимости по формуле (2) трехслойные резонансы отдельно не выделяются. Их положение найдено по суммарной частотной зависимости, на которую влияют и другие резонансы. Поэтому соответствие координат $x_{m}^{\prime}$ и $x_{m}$ следует признать хорошим. С ростом $x$ разность $\left(x_{m}^{\prime}-x_{m}\right)$ уменьшается, поскольку более короткие волны меньше «чувствуют» кривизну границы.

Как и следовало ожидать, амплитуды трехслойных резонансов имеют наибольшие значения в окрестностях толщинных резонансов, так как при выполнении условия толщинного резонанса максимальна амплитуда волны, проходящей в заполнитель. Любопытно отметить, что хотя по частотной зависимости трудно определить точное положение толщинного резонанса, его влияние проявляется в сильном росте амплитуды трехслойных резонансов по мере приближения к $x_{l}^{\prime}(8)$. Минимальные значения амплитуды частотной зависимости в окрестностях двух первых толщинных резонансов $l=1$ и $l=2$ составляют 0,109 при $m=57$ и 0,182 при $m=115$.

Резонанс волн в заполнителе. При $5 \leqslant x \leqslant 12$ на частотной зависимости видны резонансы переотраженных волн, распространяющихся в заполнителе по квадрату, периметр которого $L=4 \sqrt{2} b$.

Резонанс этих волн имеет место при условии

$$
k L=2 \pi n \quad(n=1,2,3, \ldots),
$$

которое можно переписать в виде

$$
x_{n}^{\prime}=\frac{a}{b} \frac{\pi n}{2 \sqrt{2}} .
$$

Из этого равенства легко найти шаг следования $\Delta x_{n}^{\prime}$ резонансов (максимумов) этого типа на частотной зависимости

$$
\Delta x_{n}^{\prime}=\frac{a}{b} \frac{\pi}{2 \sqrt{2}} .
$$

При $h=1 / 8$ имеем $\Delta x_{n}^{\prime}=1,269$. В табл. 2 приведены положения резонансов волн, переотражающихся по квадрату, рассчитанные по формуле (15). Для сравнения в табл. 2 внесены значения максимумов $x_{n}$, найденные по суммарной частотной зависимости (2). Небольшой почти неизменный сдвиг координат $x_{n}$ относительно $x_{n}^{\prime}$, по-видимому, связан с вкладами волн иных типов, никак не учитываемых в условии (15).

Хотя местоположение резонансов волн, переотражающихся в запол- 


\begin{tabular}{rrrr}
$n$ & & & Tаблица 2 \\
& $x^{\prime}{ }_{n}$ & $x_{n}$ & $-\left(x^{\prime}{ }_{n}-x_{n}\right)$ \\
\hline 4 & 5,08 & 5,18 & 0,10 \\
5 & 6,35 & 6,48 & 0,13 \\
6 & 7,62 & 7,75 & 0,13 \\
7 & 8,89 & 9,00 & 0,11 \\
8 & 10,16 & 10,23 & 0,07 \\
9 & 11,42 & 11,52 & 0,10
\end{tabular}

нителе по квадрату с периметром $4 \sqrt{2} b$, достаточно хорошо описывается формулой (15), углы входа-выхода этих волн являются закритическими. Тем не менее при выбранной относительной толщине $h=1 / 8$ волновая толщина $x h$ в рассматриваемом диапазоне волнового радиуса $5 \lesssim x \lesssim 12$ достаточно мала и обеспечивает прозрачность наружного слоя.

\section{3. Временная зависимость}

Для проверки работоспособности коротковолновой асимптотической формулы (6), учитывающей расходимость лучей при переотражении в цилиндрическом слое, был выполнен прямой расчет амплитуды давления, состоящей из зеркально отраженной волны и волн, переотраженных в цилиндрическом слое.

Падающий волновой пакет был принят в виде отрезка синусоиды конечной длительности

$$
\begin{gathered}
p_{i}=p_{*} \sin \left(x_{0} t_{1}\right) V\left(t_{1}\right), \quad V\left(t_{1}\right)=H\left(t_{1}\right)-H\left(t_{1}-\tau_{*}\right), \\
t_{1}=t+(r \cos \theta-1), \quad \tau_{*}=s \tau_{0}, \quad \tau_{0}=2 \pi / x_{0} .
\end{gathered}
$$

Здесь $H$ - единичная функция Хевисаида, $x_{0}$ - волновой радиус, соответствующий частоте заполнения в падающей волне, $\tau_{0}-$ период, $s-$ целое число периодов $\tau_{0}$ в пакете, $\tau_{*}$ - длительность пакета,

Тогда входящая в (1) функция $g^{F}(x)$ принимает вид

$$
g^{F}(x)=\frac{\exp \left(i z_{0}-1\right)}{x_{0}\left(y^{2}-1\right)}, \quad z_{0}=2 \pi s y, \quad y=x / x_{0} .
$$

Модуль функции $g^{F}(x)$ определяется формулой

$$
\left|g^{F}(x)\right|=\frac{\sqrt{2}}{x_{0}} \varphi\left(y, z_{0}\right), \quad \varphi\left(y, z_{0}\right)=\frac{\sqrt{1-\cos z_{0}}}{y^{2}-1} .
$$

Функция $\left|g^{F}(x)\right|$ является пернодической. Ее период составляет $s^{-1}$, а величина глобального экстремума равна $\pi s / \sqrt{2}$.

Для достижения хорошей точности при выполнении обратного преобразования Фурье по его изображению (1), (2), (18) необходимо иметь на периоде $s$ достаточное число расчетных точек

$$
1 \gg s l_{x},
$$

где $l_{x}$ - шаг счета частотной зависимости.

Чтобы отделить процесс зеркального отражения от внешней поверхности цилиндра и переотражение в наружном слое от процесса переотражения в заполнителе достаточно проанализировать временную зависимость до времен, при которых волны, переотраженные в заполнителе, просто не успевают дойти до точки наблюдения

$$
t<2\left(h \frac{c}{c_{1}}+2 \frac{b}{a} \frac{c}{c_{10}}\right) .
$$


Приняв $t=\tau_{*}$ (17) и зафиксировав $x_{0}$, найдем максймально допустимое число периодов

$$
s \leqslant \frac{x_{0}}{\pi}\left(h \frac{c}{c_{1}}+2 \frac{b}{a} \frac{c}{c_{10}}\right) .
$$

Значение $x_{0}$ выберем в окрестности первого толщинного резонанса $x_{0} \sim x_{l}(l=1)(8)$. При выбранных параметрах (4) получим $s \leqslant 60$. Назначим $s=48$. За эти 48 периодов процесс переотражения в наружном слое успевает достичь квазистационарного значения. Стационарное значение достигается только при $s \rightarrow \infty$. При выбранном $s$ период спектра $g^{F}(x)$ составляет $1 / 48$ и на него укладывается 5,33 шага счета частотной зависимости $l_{x}=1 / 256$. Шаг счета по времени $l_{t}$ выберем равным 0,004 . Тогда во временной области на один период $\tau_{0}(17)$ приходится около 16 шагов $l_{t}$.

Расчеты временных зависимостей выполнены при пяти значениях $x_{0}$, соответствующих трехслойным резонансам с индексами $m=55,57,58$, 59,61 .

На рисунке крестиками показаны найденные таким образом квазистационарные амплитуды. Они хорошо качественно и количественно согласуются с пунктирной кривой. Это означает, что коротковолновая асимптотическая формула (6) действительно хорошо описывает процесс переотражения волн в цилиндрическом слое.

Авторы выражают благодарность В. М. Корсунскому за выполнение расчетов.

\section{Л И ТЕ Р А Т Р А}

1. Векслер Н. Д. Рассеяние импульсов на упругих цилиндрах. Таллин, «Валгус», 1980.

2. Franz, W. Z. Naturforschung, 9a, 705-716 (1954).

3. Shirley, D. J., Diercks, K. J. J. Acoust. Soc. Amer., 48, № 5 (Part 2), 1275$1281(1970)$.

Институт кибернетики

Академии наук Эстонской ССР

Заочный Северо-Западный

политехнический институт
Поступила в редакцию $3 /$ I 1984

\section{N. VEKSLER, J. KARTAŠOV}

\section{AKUSTILISTE LAINETE HAJUMINE VEDELIKUGA TÄIDETUD VEDELALT RINGSILINDRILT}

Artiklis on esitatud vedelikuga täidetud vedelalt ringsilindrilt hajunud akustilise laine rōhu sagedusliku ja ajalise sõltuvuse arvutustulemused ning selgitatud hajumisprotsessi resonantset olemust. On toodud valem lainekomponentide arvutamiseks, arvestades nende korduvat peegeldumist silindrilises kihis. Arvutustulemusi on võrreldud tulemustega, mis on saadud piirolukorras, kui kiht on tasapinnaline. 


\section{SOUND WAVE SCATTERING ON A CIRCULAR FLUID CYLINDER WITH A COAXIAL FLUID FILLER}

A plane acoustical wave falls on the cylinder and is scattered by it. The acoustic pressure at backscattering in the far field is considered. The frequency dependence of the scattered pressure $\left|p^{F}(x)\right|(2)$ is shown in the Figure. Three main types of wave resonances are found. The first of them is caused by the reflection in the outer cylindrical layer, the second by the reflection along the diameter and the third by the reflection of a wave in the filler propagating on the square path. The position of the resonances is in a rather good agreement with the given one by the formula of shortwave asymptotics in the limit plane case. An approximate formula (6) is proposed to describe the specular and multiple reflected waves in the cylindrical layer. According to this formula the calculation results are shown in the Figure by the dotted line. If the divergence of the multiple reflected wave is omitted, the pressure will be described by the formula (7) (the broken line in the Figure). The calculation of the time dependences (2) were made at five values of the wave radius.

The calculation results (marked by the crosses in the Figure) are in good agreement with the results prescribed by the formula (6). 